\title{
ON UNIQUENESS OF FLOWS OF A DIPOLAR FLUID
}

\author{
by R. N. HILLS \\ (Received 30th May 1970)
}

\section{Introduction}

In 1964 Green and Rivlin (1) introduced a theory of simple force and stress multipoles founded on conventional kinematics. Using a work formula, the force and stress multipoles were defined with the help of the velocity field and its spatial derivatives. More recently, within the framework of this general study, Bleustein and Green (2) examined the theory of the simplest multipolar fluid, the dipolar fluid, and formulated constitutive equations for a homogeneous incompressible linear dipolar fluid.

In this paper, after giving a resumé of the basic field equations governing the dipolar fluid, we consider for the constitutive model proposed by Bleustein and Green, the uniqueness of flows in a bounded region. We establish that, provided the second velocity gradients remain bounded, then the solutions of the initial value problem in which the velocity and velocity gradient are prescribed on the boundary are unique. Moreover, the same can also be said for a sub-class of dipolar fluids when the velocity and dipolar tractions are prescribed on the boundary. These conclusions are based on energy considerations which are reminiscent of the familiar technique of establishing uniqueness in bounded regions for the classical Navier-Stokes theory (see Serrin (3), p. 251).

In addition to the generalization of the concepts of body and surface forces, the theory of simple dipolar continuum mechanics also generalizes the concepts of kinetic energy and inertia. In a forthcoming paper Green and Naghdi (4) discuss generally the dipolar inertia and, for the case in which the form of the kinetic energy is the same as that postulated in (2), these authors argue in favour of adopting a different form for the fluid inertia from that assumed in (2). The final section of this present paper shows that when the fluid inertia takes this new form the parallel uniqueness theorems can be established when the first and second velocity gradients remain bounded.

\section{Basic equations for a dipolar fluid}

We refer the motion of the continuum to a fixed system of rectangular Cartesian axes. Throughout the paper repeated subscripts denote summation 
over the range $1,2,3$. For an incompressible dipolar fluid the relevant field equations are (see (2))

$$
\begin{gathered}
\tau_{j i, j}-\Sigma_{(k j) i, k j}+\rho\left(b_{i}-B_{j i, j}\right)=\rho\left(\dot{v}_{i}-\Gamma_{j i, j}\right), \\
v_{k, k}=0
\end{gathered}
$$

where $\rho$ is the mass density, $v_{i}$ the velocity vector and $\Sigma_{\left(k_{j}\right) i}$ are the components of the dipolar stress $\Sigma_{k j i}$ which are symmetric in the first two indices. The symmetric tensor $\tau_{i j}$ is related to the non-symmetric monopolar stress $\sigma_{i j}$ by

$$
\tau_{i j}=\sigma_{i j}+\Sigma_{k i j, k}+\rho\left(F_{i j}-\Gamma_{i j}\right),
$$

and the dipolar inertia $\Gamma_{i j}$ adopted by Bleustein and Green (2) is

$$
\Gamma_{i j}=d^{2}\left(\dot{v}_{j, i}-v_{j, k} v_{k, i}\right) \text {, }
$$

while that proposed by Green and Naghdi (4) is

$$
\Gamma_{i j}=d^{2}\left(\dot{v}_{j, i}-v_{j, k} v_{k, i}-v_{j, k} v_{i, k}+v_{k, i} v_{k, j}\right) \text {, }
$$

where in both cases $d$ is a constant. We shall assume that the monopolar and dipolar body forces per unit mass, $b_{i}$ and $B_{i j}$ respectively, are zero. However, the present paper is easily modified to cover the situation when these forces are derivable from potentials, that is

$$
b_{i}=\Phi_{, i}, \quad B_{i j}=\Psi_{, i j}
$$

In the equations (2.1)-(2.4) a superposed dot denotes the material time derivative and a subscript $k$ following a comma indicates partial differentiation with respect to the space variable $x_{k}$. If the dipolar tractions associated with the surface whose unit normal is $n_{k}$ are $T_{i j}$ and if, in addition, $T_{i j}$ is a linear function of $n_{k}$ then

$$
T_{i j}=n_{k} \Sigma_{k i j}
$$

For a homogeneous isotropic incompressible linear dipolar fluid which is undergoing homothermal motions the constitutive equations for $\tau_{i j}$ and $\Sigma_{(i i) k}$ are (2),

$$
\begin{gathered}
\tau_{i j}+\phi \delta_{i j}=2 \mu v_{(i, j)}, \\
\Sigma_{(i j) k}+\psi_{i} \delta_{j k}+\psi_{j} \delta_{i k}=h_{1} \delta_{i j} v_{k, u}+2 h_{2} v_{(i, j), k}+h_{3} v_{k, i j}
\end{gathered}
$$

where $\delta_{i j}$ is the Kronecker delta, $\phi$ and $\psi_{i}$ are arbitrary functions that arise from the incompressibility of the fluid, $\mu, h_{1}, h_{2}, h_{3}$ are constants and

$$
v_{(i, j)}=\frac{1}{2}\left(v_{i, j}+v_{j, i}\right) \text {. }
$$

The thermodynamical restrictions imposed by the Clausius-Duhem inequality upon the coefficients appearing in the equations (2.6) and (2.7) are reported in (2) to be

$$
\begin{gathered}
\mu \geqq 0, \quad 2 h_{1}+h_{3} \geqq 0, \quad 2 h_{2}+h_{3} \geqq 0, \\
h_{3}-h_{2} \geqq 0, \quad 5 h_{1}-h_{2}+2 h_{3} \geqq 0 .
\end{gathered}
$$

We note for future reference that these inequalities imply $h_{3} \geqq 0$ and $h_{1}+h_{3} \geqq 0$. 
Irrespective of the form of the dipolar inertia, there is inherent in the theory a certain degree of non-uniqueness for, as pointed out by Green and Rivlin (1), the skew-symmetric part $\Sigma_{[i j] k}$ of $\Sigma_{i j k}$ makes no contribution to the governing equations and it is therefore arbitrary in the interior of the fluid. Furthermore, there is a non-uniqueness associated with the constraint of incompressibility for when the equations (2.5) and (2.6) are substituted in (2.1) and (2.3) a vector partial differential equation is obtained involving the velocity field and the quantity $p=\phi-2 \psi_{i, i}$ (see (2), equation 5.18). These three equations together with the equation (2.2) yield four equations for determining $v_{i}$ and $p$. However, a knowledge of the function $p$ at an interior point of the fluid is not sufficient to determine the functions $\phi$ and $\psi_{i}$ at that point. As pointed out by Hills (5) the implication of this observation is that it is not possible to state explicitly the values taken by the stresses at any interior point of the fluid.

We denote the interior of the three dimensional bounded flow domain $\Omega$ with surface $\partial \Omega$ by $\Omega^{0}$. Thus, we assume that equations (2.3)-(2.7) are valid on the product set $\Omega \times[0, T]$ where $T$ is a finite instant of time, while the equations (2.1), (2.2) and (2.8) are valid on $\Omega^{0} \times(0, T]$. We shall restrict our attention to classical solutions which are assumed to exist subject to the prescribed initial conditions

$$
v_{i}(\boldsymbol{x}, 0)=f_{i}(\boldsymbol{x}) \text { in } \Omega \times 0
$$

and prescribed boundary data of the type

or of the type

$$
\text { I }\left\{\begin{array}{cll}
\text { (i) } v_{i}(x, t)=g_{i}(x, t) & \text { on } & \partial \Omega \times[0, T] \\
\text { (ii) } n_{k} \Sigma_{k i j}=N_{i j}(x, t) & \text { on } & \partial \Omega \times[0, T] \\
\text { (iii) } f_{i}=g_{i} & \text { on } & \partial \Omega \times 0,
\end{array}\right.
$$

$$
\text { II }\left\{\begin{array}{lll}
\text { (i) } v_{i}(x, t)=g_{i}(x, t) & \text { on } & \partial \Omega \times[0, T] \\
\text { (ii) } D v_{i}(x, t)=h_{i}(x, t) & \text { on } & \partial \Omega \times[0, T] \\
\text { (iii) } f_{i}=g_{i} & \text { on } & \partial \Omega \times 0
\end{array}\right.
$$

where $n_{k}$ are the Cartesian components of the unit normal to $\partial \Omega$ and $D$ is the normal gradient operator.

\section{Uniqueness: Case 1}

In this section we shall adopt (2.4a) as the form for the dipolar inertia and say that $v_{i}$ is a solution if it satisfies (2.2), the vector partial differential equation that results from (2.1) on using (2.4a), (2.5)-(2.7), together with the designated initial condition (3.1) and either boundary data of type I or II. Moreover, the velocity is said to be of class $\mathscr{A}$ if

$$
\sup _{\mathbf{\Omega}^{\mathrm{o}} \times(0, \mathrm{~T})} v_{i, j k} v_{i, j k} \leqq N^{2}
$$

for some prescribed constant $N$. We now prove the following theorem:- 
If initial data (3.1) and boundary data of type I are prescribed then, for the sub-class of fluids for which $h_{1} \geqq 0, h_{2}=0$, the governing equations have at most one solution in the class $\mathscr{A}$.

We shall assume that there are two solutions $v_{i}$ and $v_{i}^{*}$ and on setting $w_{i}=v_{i}-v_{i}^{*}$ and utilizing the linearity of the constitutive equations (2.5) and (2.6), we obtain from (2.1) and (2.4a) by subtraction the equation

$$
\begin{aligned}
\partial_{t} w_{l}-d^{2} \partial_{t} w_{i, j j}+w_{j} v_{i, j}+v_{j}^{*} w_{i, j}-d^{2}\left(w_{k} v_{i, k j}+v_{k}^{*} w_{l, k j}\right)_{, j} & \\
& =\frac{1}{\rho}\left(\bar{\tau}_{j i, j}-\Sigma_{(k) t, k j}\right)
\end{aligned}
$$

where $\partial_{t}$ denotes partial differentiation with respect to time holding the spatial variable fixed and $\bar{\tau}_{j t}$ and $\bar{\Sigma}_{(k j) t}$ denote the stress tensors appropriate to the difference velocity $w_{i}$. Then, by forming the scalar product of (3.2) with $w_{i}$ using the incompressibility condition, it follows that

$$
\begin{aligned}
w_{i} \partial_{t} w_{i} & +d^{2} w_{i, j} \partial_{t} w_{i, j} \\
& =\frac{1}{\rho}\left(w_{i} \bar{\tau}_{j i, j}-w_{i} \bar{\Sigma}_{(k j) i, k j}\right)-w_{i} w_{j} v_{i, j}-d^{2} w_{i, j} w_{k} v_{i, k j}^{*} \\
& -\frac{1}{2}\left(w_{i} w_{i} v_{j}^{*}\right)_{, j}+d^{2}\left[w_{i} \partial_{t} w_{i, j}+w_{i}\left(w_{k} v_{i, k j}+v_{k}^{*} w_{i, k j}\right)-\frac{1}{2} v_{j} w_{i, k} w_{i, k}\right]_{, j} .
\end{aligned}
$$

Next, we introduce the function $R(t)$ defined by

$$
R(t)=\frac{1}{2} \int_{\Omega_{t}}\left(w_{i} w_{i}+d^{2} w_{i, j} w_{i, j}\right) d V
$$

where the symbol $\Omega_{t}$ indicates that the integral is to be taken over $\Omega$ at time $t$. Consequently, on using (3.3), the divergence theorem and the boundary condition $w_{i}=0$ on $\partial \Omega \times[0, T]$ we deduce

$$
\frac{d R}{d t}=\int_{\Omega_{i}}\left\{\frac{1}{\rho}\left(w_{i} \bar{\tau}_{j i, j}-w_{i} \bar{\Sigma}_{(k j) i, k j}\right)-\left(w_{i} w_{j} v_{i, j}+d^{2} w_{i, j} w_{k} v_{i, k j}^{*}\right)\right\} d V
$$

The identity

$$
w_{i} \bar{\Sigma}_{(k j) i, k j}=\left(w_{i} \bar{\Sigma}_{k j i, k}\right), j-\left(w_{i, j} \bar{\Sigma}_{k j i}\right)_{, k}+w_{i, k j} \bar{\Sigma}_{(k j) i}
$$

allows us to rewrite (3.4) as

$$
\begin{aligned}
\frac{d R}{d t}=-\int_{\Omega_{t}}\left\{\frac{1}{\rho}\left(w_{i, j} \bar{\tau}_{j i}+w_{i, j k} \bar{\Sigma}_{(k j) i}\right)\right. & \left.+\left(w_{i} w_{j} v_{i, j}+d^{2} w_{i, j} w_{k} v_{i, k j}^{*}\right)\right\} d V \\
& +\int_{\partial \Omega_{t}}\left(w_{i} n_{j} \bar{\tau}_{j i}+w_{i, j} n_{k} \bar{\Sigma}_{k j i}-w_{i} \bar{\Sigma}_{k j i, k}\right) d A
\end{aligned}
$$

and by employing the boundary conditions I we see that the surface integral 
vanishes. If we now substitute for the stress tensors using the constitutive equations (2.5) and (2.6), it follows by (2.2) that

$$
\begin{aligned}
\frac{d R}{d t}=-\int_{\Omega_{t}} \frac{1}{\rho}\left(\mu w_{(i, j)} w_{(i, j)}+h_{1} w_{i, k k} w_{i, r r}\right. & +h_{3} w_{i, j k} w_{i, j k}+2 h_{2} w_{i, j k} w_{k, j i} \\
& \left.+\rho w_{i} w_{j} v_{l, j}+\rho d^{2} w_{i, j} w_{k} v_{i, k j}^{*}\right) d V .
\end{aligned}
$$

Then, for the materials in which $h_{1} \geqq 0$ and $h_{2}=0$, since $\mu \geqq 0$ and $h_{3} \geqq 0$ we can deduce from (3.6) that

$$
\frac{d R}{d t} \leqq-\int_{\Omega_{t}}\left(w_{i} w_{j} v_{i, j}+d^{2} w_{i, j} w_{k} v_{i, k j}^{*}\right) d V .
$$

If $-m$ is the lower bound for the characteristic roots of the tensor $v_{(i, j)}$ in $\Omega^{0} \times(0, T)$ then since $v_{i, i}=0$ it follows that $m \geqq 0$ and we can use the familiar argument employed in the classical Navier-Stokes theory to bound the first term on the right-hand side of (3.7) (see Serrin (3) p. 252). Moreover we can apply the arithmetic-geometric mean and Schwarz inequalities to the second term on the right-hand side of (3.7) to deduce

$$
\frac{d R}{d t} \leqq k \int_{\Omega_{t}} w_{i} w_{i} d V+\frac{1}{2} d^{2} \int_{\Omega_{t}} w_{i, j} w_{i, j} d V
$$

where $k=m+\frac{1}{2} d^{2} N^{2}$. Consequently

$$
\frac{d R}{d t} \leqq(2 k+1) R
$$

so that by integrating from $t=0$ to $t=T$ using the initial conditions we conclude

$$
R(T) e^{-(2 k+1) T} \leqq 0 .
$$

Since $T$ was an arbitrary instant, $R(T)$ must be identically zero which in turn implies $w_{i}$ is identically zero. Hence the two flows $v_{i}$ and $v_{i}^{*}$ are identical.

We remark that in the preceding proof the boundary condition $\mathrm{I}_{(\mathrm{ii})}$ enables us to show that the surface integral

$$
\int_{\partial \Omega_{t}} \frac{1}{\rho} w_{i, j} n_{k} \bar{\Sigma}_{k j i} d A
$$

is zero. Now given any surface we can express the velocity gradient in terms of the surface gradient operator $D_{j}$ and the normal gradient operator $D$ :

$$
v_{i, j}=D_{j} v_{i}+n_{j} D v_{i}
$$

where $n_{j}$ is the unit normal to the surface. In particular for the surface $\partial \Omega_{t}$, since the velocity is specified on this surface, the first term on the right-hand side of (3.9) can be evaluated. Consequently, if we specify boundary data of 
type II, it is easily seen that $w_{i, j}=0$ on $\partial \Omega \times[0, T]$ and therefore the integral (3.8) will again vanish. Moreover, employing the identity

$$
\begin{aligned}
h_{1} w_{i, k k} w_{i, r r} & +h_{3} w_{i, j k} w_{i, j k}+2 h_{2} w_{i, j k} w_{k, j i} \\
& =\left(h_{1}+h_{3}\right) w_{i, k k} w_{i, r r}+h_{3}\left(w_{i, k} w_{i, k j}-w_{i, j} w_{i, k k}\right)_{, j}+2 h_{2}\left(w_{i, j} w_{k, j i}\right)_{, k}
\end{aligned}
$$

together with the divergence theorem and the inequality $h_{1}+h_{3} \geqq 0$, we see that if boundary data of type II is specified then (3.7) can be deduced from (3.6) independent of any restriction on $h_{1}, h_{2}, h_{3}$ other than those implied by (2.8). Hence we have established:-

If initial data (3.1) and boundary data of type II are prescribed then the governing equations have at most one solution in the class $\mathscr{A}$.

\section{Uniqueness: Case 2}

In this final section we adopt $(2.4 b)$ as the dipolar inertia so that henceforth $v_{i}$ is a solution if it satisfies (2.2), the vector partial differential equation that results from (2.1) on using (2.4b)-(2.7) together with the initial condition (3.1) and either boundary data of type I or II. Moreover, the velocity is said to be of class $\mathscr{B}$ if

$$
\sup _{\Omega^{0 \times(0, T)}} v_{i, j} v_{i, j} \leqq M^{2}, \sup _{\Omega^{0 \times(0, T)}} v_{i, j k} v_{i, j k} \leqq N^{2}
$$

for some prescribed constants $M$ and $N$. Then, corresponding to the results of the previous section, we now establish the following two theorems:-

If initial data (3.1) and boundary data of type I are prescribed then, for the sub-class of fluids for which $h_{1} \geqq 0, h_{2}=0$, the governing equations have at most one solution in the class $\mathscr{B}$.

If initial data (3.1) and boundary data of type II are prescribed then the governing equations have at most one solution in the class $\mathscr{B}$.

We need only concern ourselves with proving the first of these results since the second will follow from the first by an argument similar to the one set out in the previous section. We again assume that there are two solutions and find that the equation satisfied by the difference solution $w_{i}$ is obtained by adding the term

$$
d^{2}\left(w_{i, k} v_{j, k}+v_{i, k}^{*} w_{j, k}-w_{k, j} v_{k, i}-v_{k, j}^{*} w_{k, i}\right)_{, j}
$$

to the right hand side of (3.2). Consequently, the equation corresponding in this situation to (3.3) has the extra terms

$$
d^{2} w_{i, j} w_{i, k} v_{j, k}^{*}-d^{2} w_{i, j} w_{k, j} v_{k, i}^{*}-d^{2}\left[w_{i}\left(w_{i, k} v_{j, k}+v_{i, k}^{*} w_{j, k}-w_{k, j} v_{k, i}-v_{k, j}^{*} w_{k, i}\right)\right], j
$$

included on the left hand side. Then, by following an analysis parallel to that of Section 3 with these extra terms included, for materials in which $h_{1} \geqq 0$, $h_{2}=0$, we arrive at the inequality

$$
\frac{d R}{d t} \leqq-\int_{\Omega_{t}} w_{i} w_{j} v_{i, j} d V+d^{2} \int_{\Omega_{t}}\left(w_{i, j} w_{i, k} v_{j, k}-w_{i, j} w_{k} v_{i, k j}^{*}-w_{i, j} w_{k, j} v_{k, i}^{*}\right) d V
$$


Since the velocity is now of class $\mathscr{B}$ we can apply the arithmetic-geometric mean and Schwarz inequality to obtain

$$
\frac{d R}{d t} \leqq k_{1} \int_{\Omega_{\mathrm{t}}} w_{i} w_{i} d V+d^{2} k_{2} \int_{\Omega_{\mathrm{t}}} w_{i, j} w_{i, j} d V
$$

where $k_{1}=\left(m+\frac{1}{2} d^{2} N^{2}\right), k_{2}=\frac{1}{2}\left(3+2 M^{2}\right)$ and $m$ has the same meaning as in the previous section. Consequently,

$$
\frac{d R}{d t} \leqq 2\left(k_{1}+k_{2}\right) R
$$

and it follows directly that the difference velocity is identically zero. Therefore the theorem is proved.

\section{REFERENCES}

(1) A. E. Green and R. S. Rivlin, Simple force and stress multipoles, Arch. Rational Mech. Anal. 16 (1964), 325.

(2) J. L. Bleustern and A. E. Green, Dipolar fluids, Internat. J. Engrg. Sci. 5 (1967), 323.

(3) J. SerrIn, Handbuch der Physik, Vol. VIII/1 (Edited by S. Flügge and C. Truesdell, Springer-Verlag, 1959).

(4) A. E. Green and P. M. NAGHdi, A note on dipolar inertia. To appear.

(5) R. N. Hitls, The slow flow of a dipolar fluid past a sphere, Internat. J. Engrg. Sci. 5 (1967), 957.

THE UNIVERSITY

NEWCASTLE-UPON-TYNE 